\title{
Specific and straightforward molecular investigation of $\beta$-thalassemia mutations in the Malaysian Malays and Chinese using direct TaqMan genotyping assays
}

\author{
S.L. Kho ${ }^{1}$, K.H. Chua ${ }^{1}$, E. George ${ }^{2}$ and J.A.M.A. Tan ${ }^{1}$ \\ ${ }^{1}$ Department of Biomedical Science, Faculty of Medicine, \\ University of Malaya, Kuala Lumpur, Malaysia \\ ${ }^{2}$ Department of Pathology-Hematology, \\ Faculty of Medicine and Health Sciences, Universiti Putra Malaysia, \\ Serdang, Selangor, Malaysia \\ Corresponding author: J.A.M.A. Tan \\ E-mail: maryanne@um.edu.my
}

Genet. Mol. Res. 12 (3): 2409-2415 (2013)

Received April 14, 2012

Accepted November 10, 2012

Published February 28, 2013

DOI http://dx.doi.org/10.4238/2013.February.28.4

\begin{abstract}
Beta-thalassemia is a life-threatening inherited blood disorder. Rapid characterization of $\beta$-globin gene mutations is necessary because of the high frequency of Malaysian $\beta$-thalassemia carriers. A combination real-time polymerase chain reaction genotyping assay using TaqMan probes was developed to confirm $\beta$-globin gene mutations. In this study, primers and probes were designed to specifically identify 8 common $\beta$-thalassemia mutations in the Malaysian Malay and Chinese ethnic groups using the Primer Express software. "Blind tests" using DNA samples from healthy individuals and $\beta$-thalassemia patients with different genotypes were performed to determine the specificity and sensitivity of this newly designed assay. Our results showed $100 \%$ sensitivity and specificity for this novel assay. In conclusion, the TaqMan genotyping assay is a straightforward assay that allows detection of $\beta$-globin gene
\end{abstract}


mutations in less than $40 \mathrm{~min}$. The simplicity and reproducibility of the TaqMan genotyping assay permit its use in laboratories as a rapid and cost-effective diagnostic tool for confirmation of common $\beta$-thalassemia mutations in Malaysia.

Key words: $\beta$-thalassemia mutations; Malaysia; Malay; Chinese; TaqMan genotyping assay

\section{INTRODUCTION}

Thalassemia is the most common autosomal recessive disorder worldwide. It is a public health problem in Malaysia, as approximately $4.5 \%$ of Malaysians are $\beta$-thalassemia carriers (George and Ann, 2010). This disorder is caused by $\beta$-globin gene mutations that result in the reduction or absence of $\beta$-globin chain synthesis. Beta-thalassemia major patients require monthly blood transfusions and iron chelation therapies to ensure survival (Weatherall and Clegg, 2001). In the absence of appropriate management, affected patients typically do not survive beyond their teenage years.

More than 200 types of mutations in the $\beta$-globin gene complex have been reported (Weatherall, 2001). Fortunately, thalassemia is caused by only a handful of specific mutations in each different ethnic group or population. However, molecular characterization of thalassemia in Malaysia is challenging because of the presence of various $\beta$-globin gene mutations in this multi-racial population (Tan et al., 2004). In addition, the Malaysian indigenous populations possess their own set of specific mutations (Tan et al., 2010). The common mutations identified in the Malaysian Malay carriers are IVS1-5 (G-C), CD41/42 (-CTTT), CD19 (A-G), Poly A (AATA $\underline{A A}-A A T A \underline{G A}$ ), and IVS1-1 (G-T). In addition to these thalassemia mutations, $\mathrm{HbE}(\mathrm{G} \rightarrow \mathrm{A}$ bp substitution at $\mathrm{CD} 26)$ is the most common hemoglobin variant in the Malays (George and Ann, 2010). The common $\beta$-thalassemia mutations in the Malaysian Chinese are CD41/42 (-CTTT), IVS2-654 (C-T), -28 (A-G), CD17 (A-T), and CD71/72 (+A) (Tan et al., 2004).

Molecular characterization for genetic disorders is not only essential for large-scale screening of populations but also critical for prenatal diagnosis to determine whether the fetus is normal, a carrier, or affected. Prenatal diagnosis allows for effective genetic counseling and, more importantly, aids parents in making informed decisions with regard to the outcome of an affected pregnancy.

Various polymerase chain reaction (PCR) techniques, including the amplification refractory mutation system (ARMS) and reverse dot blot assays, are currently used for molecular characterization of thalassemia mutations (Tan et al., 2006). However, both techniques are labor intensive and technically demanding, and require staff to be trained in molecular techniques necessary for performing the assays and analyzing the results. Genomic sequencing is another approach that directly identifies mutations.

Molecular analysis of gene mutations has become less technically demanding with the introduction of real-time PCR. This technique has reduced diagnosis time with its simple setup procedures and the elimination of post-PCR sample processing. The TaqMan probe was originally conceived for quantitative PCR analysis that visualizes the amplification of PCR products at each cycle. It has since been applied to single nucleotide polymorphism genotyp- 
ing and mutation detection, including single base pair substitutions, insertions, and deletions (Heid et al., 1996).

In this study, a TaqMan genotyping assay was developed to detect 8 common $\beta$-thalassemia mutations in Malaysia. The common mutations were selected based on results from a molecular analysis of $616 \beta$-thalassemia and/or $\mathrm{HbE}$ families in our laboratory. The selected mutations included IVS1-5 (G-C), Poly A (AATA $\underline{A} A-A A T A \underline{G A}$ ), IVS1-1 (G-T) and $\mathrm{HbE}$ (CD26; G-A) common to the Malays, and CD41/42 (-CTTT), IVS2-654 (C-T), -28 (A$\mathrm{G})$, and CD17 (A-T) common to the Chinese.

\section{MATERIAL AND METHODS}

\section{DNA samples}

One hundred and seventy-six previously characterized DNA samples were used to develop our in-house TaqMan genotyping assay. Seventeen DNA samples were obtained from patients who were compound heterozygous, whereas the remaining 159 samples were obtained from patients homozygous for the $\beta$-thalassemia mutations. All of the samples were part of the stock DNA collection used in previous molecular analyses, and prenatal diagnosis of thalassemia carried out in the University Malaya Medical Center (UMMC) from 1995 to 2012. Informed consent was obtained from all patients before blood collection.

DNA was extracted by a simplified DNA extraction method based on the protocol as reported previously (Tan et al., 2004). The concentration of DNA was determined using a nanophotometer. All DNA samples were diluted to $20 \mathrm{ng} / \mu \mathrm{L}$ and were used as templates for real-time PCR. The $\beta$-globin gene mutations of all DNA samples from $\beta$-thalassemia patients were characterized using ARMS and genomic sequencing (Tan et al., 2010).

\section{Primer and probe design}

Eight pairs of primers were designed to amplify the region in which the 8 common mutations are located. One pair of primers was required to amplify the selected region, whereas 2 probes were required for each mutation, the first complementary to the normal DNA sequence and the other complementary to the mutant DNA sequence (Twyman, 2005). Primers and probes were designed with the aid of the Primer Express software (Applied Biosystems, Foster City, CA, USA). The primers and probes used for the TaqMan real-time PCR assay are listed in Table 1.

\section{TaqMan genotyping assay}

The PCRs were performed using an Applied Biosystems 7500 Fast Real-Time thermal cycler. The final reaction mixture $(10 \mu \mathrm{L})$ contained $1 \mu \mathrm{LDNA}$ sample, $5 \mu \mathrm{L}$ TaqMan GTXpress ${ }^{\mathrm{TM}}$ Master Mix, $0.5 \mu \mathrm{L}$ 20X TaqMan SNP Genotyping Assay, and 3.5 $\mu \mathrm{L}$ double-distilled water. The thermal cycling conditions were $95^{\circ} \mathrm{C}$ for $20 \mathrm{~s}$, followed by 40 cycles of denaturation at $95^{\circ} \mathrm{C}$ for $3 \mathrm{~s}$, and annealing/extension at $60^{\circ} \mathrm{C}$ for $30 \mathrm{~s}$. TaqMan real-time PCR assays for all 8 mutations, IVS1-5, Poly A, IVS1-1, HbE (CD26), CD41/42, IVS2-654, -28, and CD17, were performed simultaneously in different panels, and the results are shown in Figure 1. 
Table 1. Primer and TaqMan probe sequences for the 8 common mutations in Malaysia.

\begin{tabular}{|c|c|c|c|c|c|c|}
\hline \multirow[t]{2}{*}{ Mutations } & \multicolumn{2}{|c|}{ Mutation incidence (\%) } & \multirow{2}{*}{$\begin{array}{l}\text { Forward primers } \\
\left(5^{\prime}-3^{\prime}\right)\end{array}$} & \multirow{2}{*}{$\begin{array}{l}\text { Reverse primers } \\
\left(5^{\prime}-3^{\prime}\right)\end{array}$} & \multirow{2}{*}{$\begin{array}{l}\text { Normal probe } 1 \\
\left(5^{\prime}-3^{\prime}\right)\end{array}$} & \multirow{2}{*}{$\begin{array}{l}\text { Mutant probe } 2 \\
\left(5^{\prime}-3^{\prime}\right)\end{array}$} \\
\hline & Malays & Chinese & & & & \\
\hline \multirow[t]{2}{*}{ CD41/42 (-CTTT) } & 6.5 & 38.9 & CTGGTGGTCTA & CAGCATCAGG & VIC-CAGAGGTTCT & FAM-AGAGGTTGA \\
\hline & & & CCCTTGG & AGTGGACAGAT & TTGAGT-MGB-NFQ & GTCC-MGB-NFQ \\
\hline \multirow[t]{2}{*}{$\mathrm{HbE}, \mathrm{CD} 26$ (G-A) } & 33.2 & 3.3 & CAAGGTGAGC & TCTCCTTAAACCT & VIC-GGTGGTGAGG & FAM-TGGTGGTAAG \\
\hline & & & GTGGATGA & GTCTTGTA ACC & CCC-MGB-NFQ & GCCC-MGB-NFQ \\
\hline \multirow[t]{2}{*}{ IVS2-654 (C-T) } & 1.9 & 30.0 & CATGCCTCTTT & ССТСТTACATCA & FAM-TGGGTTAAGG & VIC-TTTCTGGGTTA \\
\hline & & & GCACCATTCTAA & $\begin{array}{l}\text { GTTACAATTTA } \\
\text { TATGCAGAAA }\end{array}$ & CAATA-MGB-NFQ & AGGTAATA-MGB-NFQ \\
\hline \multirow[t]{2}{*}{ IVS1-5 (G-C) } & 27.1 & 0.2 & GTGAACGTGG & CCCAGTTTCTAT & VIC-AGGTTGGTATC & FAM-AGGTTGCTA \\
\hline & & & ATGAAGTTGG & TGGTCTCCTTA & AAG-MGB-NFQ & TCAAG-MGB-NFQ \\
\hline \multirow[t]{2}{*}{$-28(A-G)$} & 0.5 & 10.8 & CAGGGAGGGC & TTGTGTCAGAAG & VIC-CCTGACTTTT & FAM-CTGACTTCT \\
\hline & & & AGGA & CAAATGTAAGC & ATGCC-MGB-NFQ & ATGCC-MGB-NFQ \\
\hline \multirow[t]{2}{*}{$\mathrm{CD} 17$ (A-T) } & 0.9 & 8.3 & AGGAGAAGTC & GCCTCACCAC & VIC-ACGTTCACCT & FAM-CGTTCACCTA \\
\hline & & & TGCCGTTACT & CAACTTCA & TGCCCC-MGB-NFQ & GCCCC-MGB-NFQ \\
\hline \multirow[t]{2}{*}{ IVS1-1 (G-T) } & 4.7 & 0.8 & GTGAACGTGG & CCCAGTTTCTA & VIC-TGGGCAGGTT & FAM-TGGGCAGTT \\
\hline & & & ATGAAGTTGG & TTGGTCTCCTTA & GGTA-MGB-NFQ & TGTAT-MGB-NFQ \\
\hline \multirow{3}{*}{ Poly A (AATAAAA-AATAGA) } & ) 2.3 & 0.0 & GGCCTTGAGC & ССАСАТТСССТТ & FAM-GCCTAATAA & VIC-TGCCTAATAGA \\
\hline & & & ATCTGGAT & TTTAGTAAAATA & AAAAC-MGB-NFQ & AAAC-MGB-NFQ \\
\hline & & & & TTCAGAAATAA & & \\
\hline
\end{tabular}

FAM = 6-carboxyfluorescein; VIC = 2'-chloro-7'-phenyl-1,4-dichloro-6-carboxyfluorescein.

\section{Evaluation of TaqMan genotyping assays}

Two hundred and twenty DNA samples from normal individuals and previously characterized $\beta$-thalassemia patients were randomly selected to evaluate the sensitivity and specificity of our newly developed in-house TaqMan genotyping assay. The genotypes of the 220 DNA samples were withheld during the molecular evaluation process.

\section{RESULTS}

Amplification plots, allelic discrimination plots and multi-component plots were generated by the 7500 version 2.5 software (Applied Biosystems) to distinguish the wild-type, heterozygous, and homozygous DNA samples. All samples were analyzed by the TaqMan Genotyper software (Applied Biosystems) by plotting the normalized intensity of reporter dyes in a scatter plot (Figure 1). Non-template controls and non-amplifiable samples were removed from the analysis.

\section{Evaluation}

The sensitivity and specificity of the TaqMan genotyping assays were calculated. Sensitivity is defined as the ability of a test to positively and correctly detect those with a disease. Specificity refers to the ability of the test to accurately detect individuals without the disease state (Indrayan, 2008). Both the sensitivity and specificity of our in-house TaqMan genotyping assay were $100 \%$. 
A

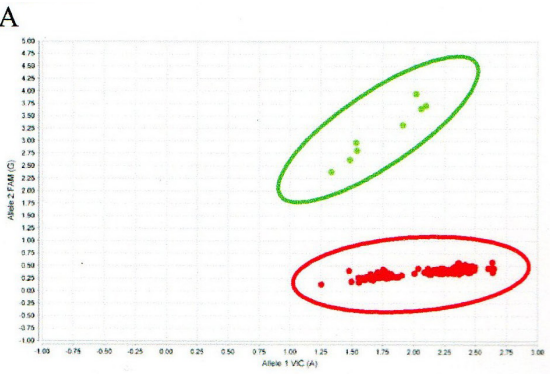

C

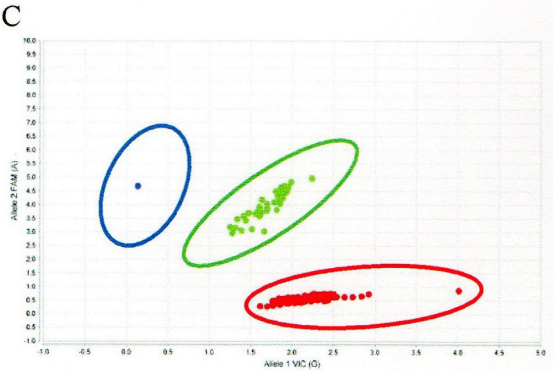

E

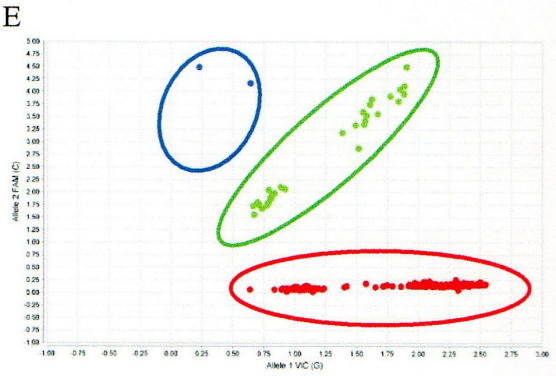

G

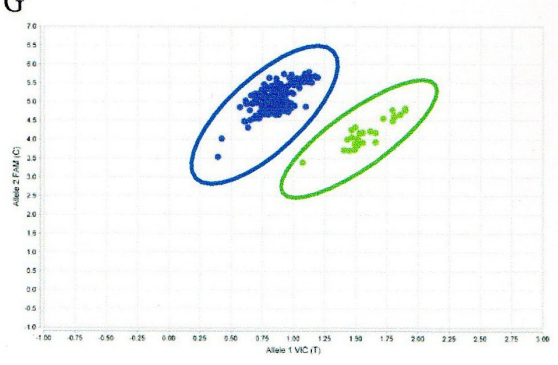

B

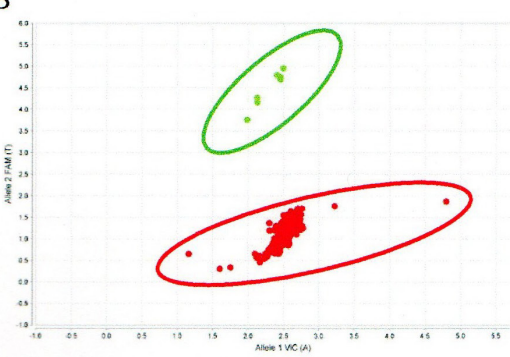

$\mathrm{D}$

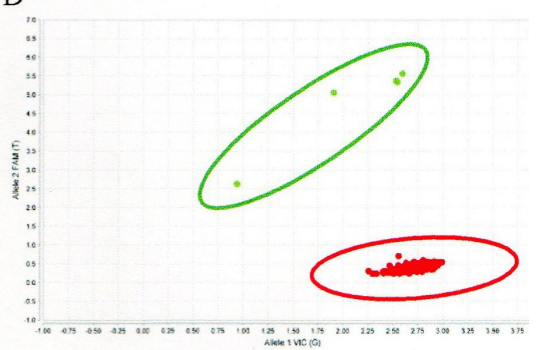

F

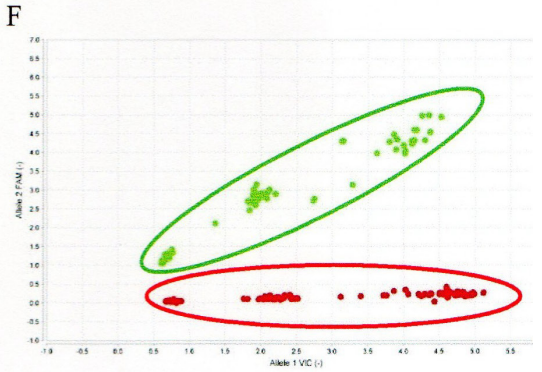

$\mathrm{H}$

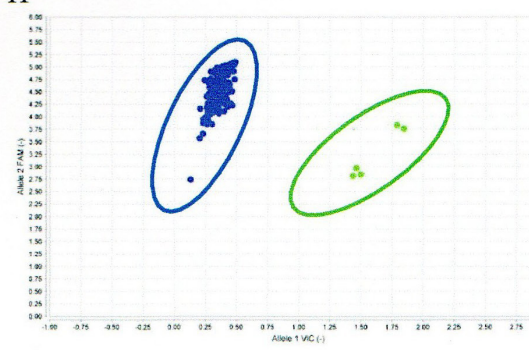

Figure 1. Scatter plots of TaqMan assays of the $8 \beta$-thalassemia mutations with 2'-chloro-7'-phenyl-1,4-dichloro-6carboxyfluorescein dye normalized fluorescence at $\mathrm{x}$-axis and 6-carboxyfluorescein dye normalized fluorescence at y-axis: A. -28, 8 heterozygote carriers (green) and 211 wild type (red); B. CD17, 7 heterozygote carriers (green) and 212 wild type (red); C. CD26, 1 homozygote (blue), 42 heterozygote carriers (green), and 176 wild type (red); D. IVS1-1, 6 heterozygote carriers (green) and 213 wild type (red); E. IVS1-5, 2 homozygote (blue), 31 heterozygote carriers (green), and 186 wild type (red); F. CD41/42, 61 heterozygote carriers (green) and 158 wild type (red); G. IVS2-654, 26 heterozygote carriers (green) and 193 wild type (blue); H. Poly A, 5 heterozygote carriers (green) and 214 wild type (blue). 


\section{DISCUSSION}

TaqMan-based genotyping assays for $\beta$-thalassemia mutation detection were successfully developed in this study. The principle of the TaqMan probe is based on fluorescence resonance energy transfer hybridization of the probe and the 5'-nuclease assay (Holland et al., 1991). The probe with 5'-fluorescence reporter dye and 3'-quencher dye anneals to target DNA between the forward and reverse primers. During amplification, the 5'-to-3' exonuclease activity of Taq DNA polymerase cleaves the nucleotides that quench fluorescence at the end of polymerization, thereby inhibiting fluorescence. The fluorescent spectra of 96 wells are continuously measured during the amplification (Heid et al., 1996). Two sets of primers (forward and reverse) and fluorescent probes are designed so that each contains nucleotides complementary to wild-type and mutant alleles. Using this principle, wild-type, heterozygous (carrier), and homozygous samples are clearly and accurately confirmed.

Reporter dyes, VIC (2'-chloro-7'-phenyl-1,4-dichloro-6-carboxyfluorescein) or FAM (6-carboxyfluorescein), which emit fluorescent light in different spectra, are designed to bind the 5'-end of probes (Twyman, 2005). At the 3'-end, the probe is coupled with the quencher (MGB-NFQ: the minor groove binder to stabilize annealing and the non-fluorescent quencher in order to act as energy transfer acceptor) (Afonina et al., 1997).

An analogous TaqMan assay was developed and reported to be effective for detection of the Sardinian Wilson's disease mutations (Zappu et al., 2010). In the present study, primers and probes were specifically designed to detect 8 common mutations in the Malaysian Malays and Chinese, which allowed for systematic detection of the common mutations found in these ethnic groups.

Quantitative real-time PCR is a closed-tube system in which post-PCR handling has been eliminated. This reduces the probability of cross-contamination from different samples. The removal of post-PCR sample handling by agarose gel electrophoresis also allows for a safer technique in which ethidium bromide, a hazardous and mutagenic chemical, is avoided.

Optimization of the TaqMan real-time PCR assay is less demanding when compared to that of ARMS, which involves manipulation of annealing temperatures and primer concentrations, magnesium chloride and/or dimethyl sulfoxide to ensure that only target sequences are amplified (Chan et al., 2001). In the TaqMan genotyping assay, only 1 reaction is required for confirmation of heterozygosity or homozygosity in patient DNA samples. In ARMS, 2 reactions are required to confirm the genotype of an individual. The entire process, from setting up the TaqMan genotyping assay to performing data analysis, can be completed within $1 \mathrm{~h}$ when compared with $4 \mathrm{~h}$ for ARMS (since ARMS requires post-PCR processing). Thus, the TaqMan genotyping assay is suitable for large-scale screening and high-throughput analysis, as it is reliable, rapid, and cost effective. In addition, the use of minute DNA concentrations $(20 \mathrm{ng} / \mu \mathrm{L})$ in the TaqMan genotyping assay is a preferable condition for prenatal diagnosis because of the limited amount of DNA that is extracted from chorionic villus samples.

\section{ACKNOWLEDGMENTS}

Research supported by the Exploratory Research Grant Scheme (ERGS \#ER0042011A), the Mosti Science Fund (\#12-02-03-2090), and the Fundamental Research Grant Scheme (FRGS/1/2012 SG06/UPM). 


\section{REFERENCES}

Afonina I, Zivarts M, Kutyavin I, Lukhtanov E, et al. (1997). Efficient priming of PCR with short oligonucleotides conjugated to a minor groove binder. Nucleic Acids Res. 25: 2657-2660.

Chan YF, Tan KL, Wong YC, Wee YC, et al. (2001). The use of the amplification refractory mutation system (arms) in the detection of rare $\beta$-thalassemia mutations in the Malays and Chinese in Malaysia. Southeast Asian J. Trop. Med. Public Health 32: 872-879.

George E and Ann TJ (2010). Genotype-phenotype diversity of $\beta$-thalassemia in Malaysia: treatment options and emerging therapies. Med. J. Malaysia 65: 256-260.

Heid CA, Stevens J, Livak KJ and Williams PM (1996). Real time quantitative PCR. Genome Res 6: 986-994.

Holland PM, Abramson RD, Watson R and Gelfand DH (1991). Detection of specific polymerase chain reaction product by utilizing the $5^{\prime} \rightarrow 3^{\prime}$ exonuclease activity of Thermus aquaticus DNA polymerase. Proc. Natl. Acad. Sci. U. S. A. 88: 7276-7280.

Indrayan A (2008). Basic Methods of Medical Research. AITBS Publisher, New Delhi.

Tan JA, George E, Tan KL, Chow T, et al. (2004). Molecular defects in the $\beta$-globin gene identified in different ethnic groups/populations during prenatal diagnosis for $\beta$-thalassemia: a Malaysian experience. Clin. Exp. Med. 4: 142-147.

Tan JA, Chin PS, Wong YC, Tan KL, et al. (2006). Characterisation and confirmation of rare $\beta$-thalassaemia mutations in the Malay, Chinese and Indian ethnic groups in Malaysia. Pathology 38: 437-441.

Tan JA, Lee PC, Wee YC, Tan KL, et al. (2010). High prevalence of alpha- and beta-thalassemia in the Kadazandusuns in East Malaysia: challenges in providing effective health care for an indigenous group. J. Biomed. Biotechnol. Doi: $10.1155 / 2010 / 706872$.

Twyman RM (2005). Single Nucleotide Polymorphism (SNP) Genotyping Techniques. Encyclopedia Diagn. Genomics Proteomics 1202-1207.

Weatherall DJ (2001). Phenotype-genotype relationships in monogenic disease: lessons from the thalassaemias. Nat. Rev. Genet. 2: 245-255.

Weatherall DJ and Clegg JB (2001). The Thalassaemia Syndromes. Blackwell Scientific, Oxford.

Zappu A, Lepori MB, Incollu S, Noli MC, et al. (2010). Development of TaqMan allelic specific discrimination assay for detection of the most common Sardinian Wilson's disease mutations. Implications for genetic screening. Mol. Cell. Probes 24: 233-235. 